\title{
«Hydrométrie 2017 : mesures et incertitudes » Bilan sur le colloque des 14 et 15 mars 2017 à Villeurbanne
}

\author{
Michel LANG ${ }^{1}$ et Jérôme Le COZ
}

\author{
${ }^{1}$ Hydrologue \\ ${ }^{2}$ Hydraulicien \\ Irstea (Lyon-Villeurbanne)
}

Cette nouvelle édition du colloque SHF Hydrométrie, après celles de 1989, 2008 et 2013, a permis de faire le point sur les avancées techniques dans le domaine de la mesure des débits des cours d'eau et dans les réseaux d'assainissement. Elle a bénéficié d'un bon niveau de participation, avec 180 inscrits, en majorité français mais aussi étrangers (Belgique, Luxembourg, Italie, Portugal, Suisse, République Démocratique du Congo, Canada, Costa Rica, Mexique, Haïti). L'origine professionnelle des participants a couvert les principaux domaines concernés : services hydrométriques de l'Etat (24\%), grands opérateurs hydrauliques (EDF, CNR, CACG, SCP - France ; CVA - Italie ; EDP - Portugal ; Hydro-Québec - Canada ; ICE - Costa Rica : 24\%), universités et organismes de recherche $(15 \%)$, bureaux d'étude (9\%), fabricants de matériels et diffuseurs de logiciels (8\%), organismes publics $(6 \%)$, autres (CACG, Société du Canal de Provence, Agence de l'Eau, EPTB, Syndicats, Conseils Départementaux, SNCF, Suez, Véolia... : 10\%), SHF (3\%). On notera la contribution de l'hydrométrie urbaine qui a diversifié les points de vue sur des problèmes en bonne partie partagés.

Cette édition 2017 a été structurée en trois thèmes : 1/ enjeux liés aux réseaux de mesure (évolutions récentes, expériences internationales) ; $2 /$ mesure et méthodes en rivières et réseaux urbains (diversité et innovations technologiques) ; 3/ qualification et valorisation des données, analyses d'incertitude. Elle a permis d'avoir des témoignages sur l'organisation de l'hydrométrie à l'international, avec des éléments de comparaison sur la gestion des réseaux en Wallonie, en Afrique sub-saharienne et en Nouvelle-Zélande, et la présentation de deux posters sur le suivi des crues éclair au Mexique et la mesure du transport solide dans le bief maritime du Congo (République Démocratique du Congo). Plusieurs communications ont porté sur des chantiers pilotés par le Ministère chargé de l'Ecologie : 1/ la ré-édition de la "Charte qualité de l'Hydrométrie ", diffusée en séance aux participants et qui actualise utilement l'édition précédente de 1998 ; 2/ l'avancement des développements autour de la banque HYDRO3, avec la modernisation du système d'information et de nouvelles fonctionnalités ; 3/ la publication d'un guide sur l'inter-comparaison des mesures de débit en rivière ; 4/ l'implication française dans les activités de normalisation autour de l'hydrométrie ; 5/ le lancement en 2015 d'une action nationale sur la sauvegarde des données limnimétriques et pluviométriques anciennes (poster).

Sans reprendre dans le détail l'ensemble des exposés et posters présentés pendant ces deux journées, on peut relever la diversité des thèmes abordés : $1 /$ des exemples d'adaptation de la configuration des seuils de contrôle aux nouvelles contraintes environnementales liées à la continuité écologique (conciliation d'une bonne sensibilité des stations hydrométriques avec le libre passage des poissons dans la rivière) ; $2 /$ des témoignages sur l'activité métrologique dans les réseaux d'assainissement, à croiser avec ce qui est fait sur le réseau hydrométrique ; 3/ 1'apport de la modélisation hydraulique pour l'extrapolation des courbes de tarage en crue et la connaissance du champ de vitesses dans une section d'écoulement ; 4/ l'utilisation des nouvelles techniques d'analyse d'images pour reconstituer le champ de vitesses de surface et in fine le débit ; 5/ l'hydrométrie sous influence maritime ou d'ouvrage hydraulique (jaugeage et courbe de tarage multi-échelles, stations vélocimétriques) ; 6/ la qualification des incertitudes des jaugeages, des courbes de tarage et des chroniques finales de débit.

Le prix du meilleur poster (parmi les 29 posters) a été attribué par le vote des participants à Aurélien Despax et al. sur "l'estimation de l'incertitude des jaugeages ADCP : résultats préliminaires des essais interlaboratoires de Chauvan 2016 ». Il vient récompenser une initiative intéressante de la communauté hydrométrique française depuis une dizaine d'années, consistant à réaliser régulièrement des campagnes de mesure regroupant de nombreuses équipes de terrain pour mieux estimer les incertitudes de mesure. La campagne de 2016 était plus particulièrement consacrée aux jaugeages ADCP et aux incertitudes liées à la configuration de la section de mesure. Les posters arrivés en deuxième et troisième position étaient ceux présentés par EDF/DTG et par la DAVAR en Nouvelle-Calédonie, respectivement sur les jaugeages par exploration du champ des vitesses par courantomètre (intérêt de rajouter des verticales intercalaires bathymétriques ; bilan sur l'étalonnage et vérification des moulinets), et sur les échelles de crue CalédoMax, un dispositif original très astucieux pour enregistrer de façon fiable et économique les niveaux maximum d'inondation.

Le grand prix de la SHF a été attribué à Rémy Garçon (EDF/DTG) pour l'ensemble de son activité en hydrologie opérationnelle, avec des contributions significatives dans le domaine de la modélisation hydrologique (modèle MORDOR) et la prédétermination des extrêmes (méthode SCHADEX), et une forte implication pour le rapprochement des connaissances en météorologie et en hydrologie. Rémy Garçon a acquis au cours de sa carrière une grande expérience qu'il a su mettre au service de son organisme (EDF) et plus généralement de la communauté hydrologique via sa participation régulière aux instances de la SHF (bureau du comité scientifique et technique, comités de pilotage de colloques, jury du prix Henri Milon).

Cette édition 2017 a permis de constater la richesse du savoir-faire de la communauté de l'hydrométrie francophone et son dynamisme, qui peuvent être valorisés lors 
de l'établissement de nouvelles normes en hydrométrie ou d'actions de coopération internationale. Les présentations ont montré l'intérêt d'approches pluridisciplinaires pour progresser dans la connaissance et la pratique de terrain, avec les complémentarités entre hydrologie, hydraulique et hydrométrie, les échanges d'expérience entre hydromètres des champs et hydromètres des villes, le croisement des données pour la qualité et la quantité des eaux.

$\mathrm{Au}$ chapitre des remerciements, nous mentionnons : l'équipe de la SHF pour l'important travail d'organisation et de logistique ; l'INSA pour l'accueil du colloque dans ses bâtiments ; la forte implication des membres du comité d'organisation qui ont relu en détail l'ensemble des résumés et articles présentés à la conférence ; nos trois présidents de session, Anne-Catherine Favre (Université Grenoble Alpes), Michel Esteves (IGE) et Christophe Brachet (OIEau) pour leur animation des discussions et le respect des horaires ; le soutien du Ministère chargé de l'Ecologie, d'EDF et de la CNR ; l'aide de doctorants et stagiaires pour la présentation des exposés et la circulation des micros dans la salle ; et les huit exposants de matériel hydrométrique présents à la conférence.

Pour la suite de cette édition, nous mentionnerons que les supports de présentation et les posters sont mis à disposition sur le site de la SHF, avec une captation vidéo des communications (sous réserve d'autorisation des auteurs). Une sélection d'articles scientifiques et technique est publiée à partir de ce numéro dans la Houille Blanche. Le Groupe Doppler Hydrométrie (GDH, https://gdh.irstea.fr), qui regroupe plus de 300 abonnés, constitue dorénavant la section Hydrométrie de la SHF. Un rythme pseudo-quadriennal semble s'être instauré, entre les éditions de 2008, 2013 et 2017 du colloque SHF en Hydrométrie. Rendez-vous à la prochaine édition en 2021 ?

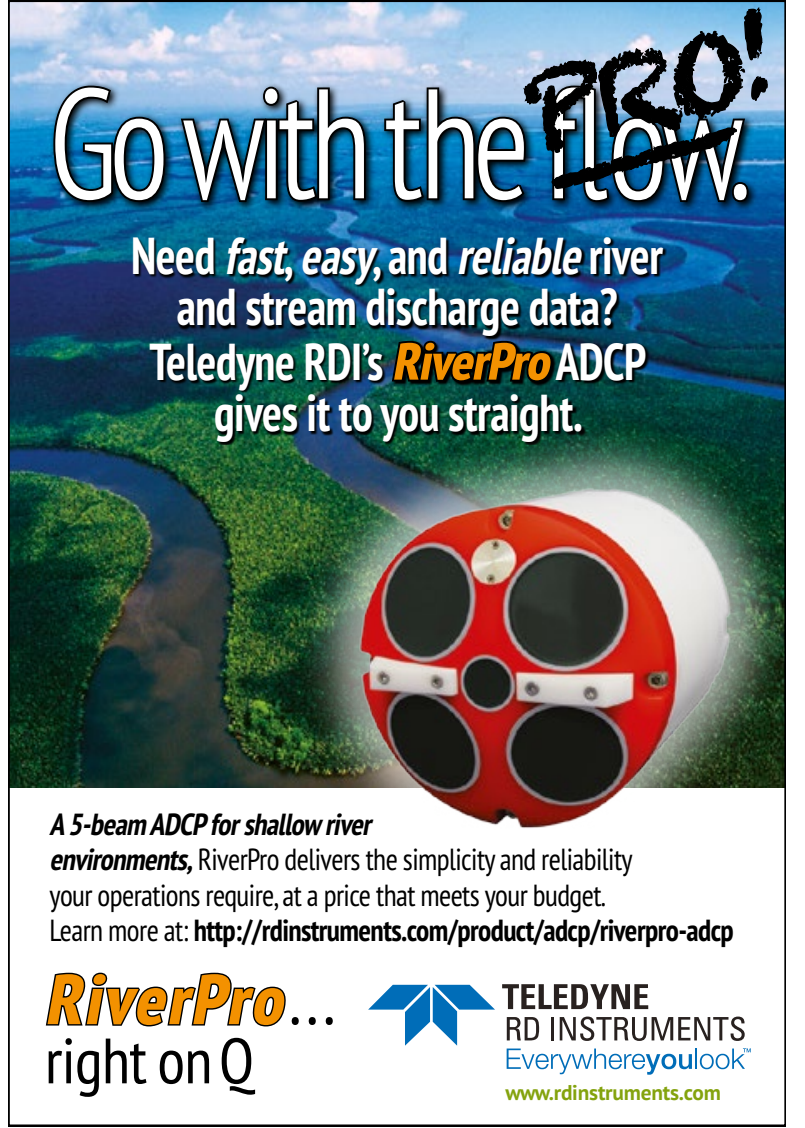

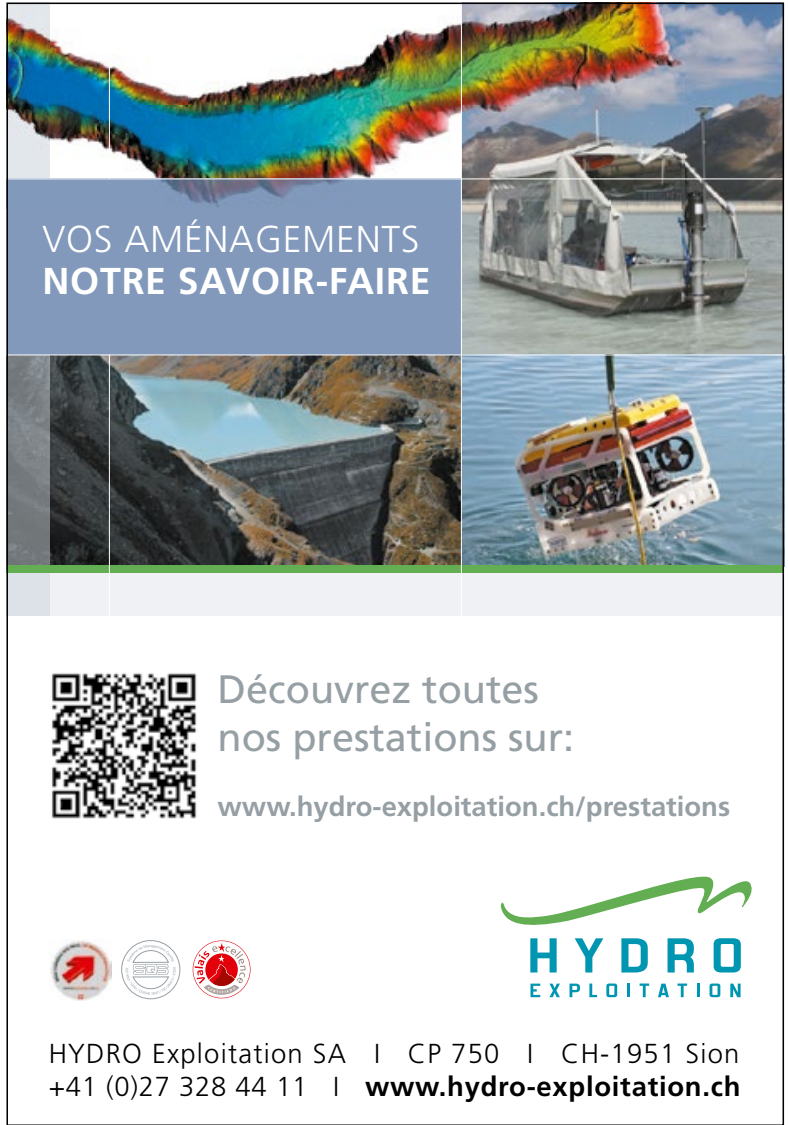

